\title{
Noticias importantes de Hispanoamérica
}

(A cargo de Alfredo A. Roggiano, con la colaboración de Horacio J. Becco [Argentina], Luis Leal [México] y Raúl Silva Castro [Chile]. El próximos números será ampliada con la contribución de colaboradores de los demás países de Iberoamérica).

INSTITUTO INTERNACIONAL DE LITERATURA IBEROAMERICANA

VIII ${ }^{\circ}$ CONGRESO

Tendrá Iugar en la Universidad de Puerto Rico, los días 26, 27 y 28 de agosto de 1957, bajo el auspicio del Gobierno y de la Universidad de San Juan de Puerto Rico.

Tema: Las literaturas del Caribe y de los paises adyacentes.

\section{Programa}

Miércoles, 28 de agosto: Por la mañana, Sesión inaugural, en La Fortaleza, San Juan. Vermouth de honor ofrecido por el Sr. Gobernador del Estado Libre Asociado de Puerto Rico, Hon. Luis Muñoz Marín.

2 p. m. Primera sesión de trabajo. Salón Jerónimo, Hotel Caribe Hilton. Presidente de la sesión: Marcel Bataillon, College de France.

1. Margot Arce, Universidad de Puerto Rico: "El mito del Paraíso en la poesía puertorriqueña".

Relator: Salvador Dinamarca, Brooklyn College.

2. Muna Lee, "Lo puertorriqueño en las Elegias de Varones Ilustres". Relator: María Teresa Babín, New York University.

3. Concha Meléndez, Universidad de Puerto Rico. "El cuento puertorriqueño y el cubano. Reflexiones sobre dos antologías".

Relator: Seymour Menton, University of Kansas.

4. Entique Anderson Imbert, University of Michigan, "La crítica literaria en el Caribe".

Relator: Cesáreo Rosa-Nieves, Universidad de Puerto Rico. 
4 p. m. Segunda sesión de trabajo. Salón Jerónimo. Hotel Caribe Hilın. Presidente de la sesión: William Atkinson, University of Glasgow.

1. Luis Monguió, University of California, Berkeley. "El negro en algunos poetas españoles y americanos anteriores a $1800^{\prime \prime}$.

Relator: Renato Rosaldo,-University of Arizona.

2. Eugenio Chang-Rodríguez, University of Pennsylvania, "La poesía negrista y la poesía de las Antillas".

Relator: Angel Luis Morales, Universidad de Puerto Rico.

3. John E. Englekirk, Tulane University, New Orleans, "El Teatro y el pueblo en el Caribe".

4. Raimundo Lazo, Universidad de La Habana, "Existencia, personalidad y expresión literaria de una Hispanoamérica insular".

Relator: Francisco Monterde, Universidad Nacional de México.

Jueves, 29 de agosto: 9.30 a.m. Tercera sesión de trabajo. Paraninfo, Universidad de Puerto Rico, Rio Piedras. Presidente de la sesión: John Van Horne, University of Illinois.

1. G. Arnold Chapman, University of California, Berkeley, "De las fuentes de Heredia".

Relator: José Arce, Dartmouth College.

2. José de Onís, University of Colorado, "William Cullen Bryant y José María Heredia. Vieja y nueva polémica".

Relator: José Ferrer Canales, Texas Southern University.

3. Arturo Torres-Rioseco, University of California, Berkeley, "Estilo poético de José Martí".

Relator: Eugenio Florit, Barnard College, Columbia University.

4. Alfredo Roggiano, State University of Iowa, "Pedro Henríquez Ureña o el pensamiento integrador".

Relator: Hugo Rodríguez Alcalá, Rutgers University.

11.30 a. m. Cuarta sesión de trabajo. Paraninfo, Universidad de Puerto Rico. Presidente de la sesión: Julio Jiménez Rueda, Universidad Nacional de México.

1. Juan Marín, Unión Panamericana, Washington, D. C., "Vida y obra de José Batres Montúfar".

Relaton: Ruth S. Lamb, Scripps College, Claremont, California.

2. Fernando Alegría, University of California, Berkeley, "Miguel Ángel Asturias, novelista del viejo y del nuevo mundo".

3. Rogelio Sinán, Secretario de Ia Embajada de Panamá en México, "Rutas de la novela panameña".

$12: 45$ p. m. Almuerzo.

3:00 p. m. Quinta sesión de trabajo. Paraninfo, Universidad de Puerto Rico. Presidente de la sesión: Ronald Hilton, Stanford University.

1. Marcel Bataillon, College de France, "La raíz colonial y criolla de las literaturas hispanoamericanas".

Relator: J. Jiménez Rueda, Universidad Nacional de México.

2. J. Riis Owre, University of Miami, "La fauna en la novela hispanoame. ricana". 
Relator: Arnulfo Trejo, U. C. L. A.

3. Max Henríquez Ureña, "Sobre las tres Antillas Mayores". Relator: George Schade, University of Texas, Austin.

4. Hugo Rodríguez Alcalá, Rutgers University, "Los sonetos de Alejandro Korn".

Viernes, 30 de agosto: 9:30 a. m. Sexta sesión de trabajo, Universidad Interamericana, San Germán. Presidente de la sesión: Jefferson Rea Spell, University of Texas.

1. Cecilia Meireles, "Sobre o folklore brasileiro". Relator: Daniel Wogan, Tulane University.

2. Reyes Carbonell, Duquesne University, "Poetas iberoamericanos: Arturo Torres-Rioseco".

Relator: Enrique A. Laguerre, Universidad de Puerto Rico.

3. Alfonso Escudero, Universidad Católica de Chile, "Hombres de tierra cálida en el clima de Chile".

Relator: Francisco Aguilera, Hispanic Foundation, Library of Congress, Washington, D. C.

2:00 p.m.: Séptima sesión de trabajo. Universidad Interamericana, San Ger. mán. Presidente de la sesión: J. de Onís, University of Colorado.

1. Allen W. Phillips, University of Chicago, "López Velarde y su concepto de la poesía en el postmodernismo".

2. Frank Dauster, Rutgers University, "Aspectos del paisaje en la poesia de Carlos Pellicer".

3. José Vázquez Amaral, Rutgers University, "Novelística de Agustín Yáñez".

Sábado, 31 de agosto: Por la mañana, Sesión de clausura del $8^{\circ}$ Congreso. San Juan.

Informe de Comisiones.

Palabras de despedida por el Subsecretario de Estado, Dr. A. Morales Carrión.

Trabajos leídos solamente por título:

José J. Arrom, Yale University, "El descubrimiento de América narrado por un humanista del Renacimiento, Fernán Pérez de Oliva".

Salvador Dinamarca, Brooklyn College, "Gabriela Mistral y su obra poética". José Ferrer Canales, Texas Southern University, "Huellas de José de Diego". Rico".

Enrique Laguerre, Universidad de Puerto Rico, "El modernismo en Puerto

Ruth S. Lamb, Scripps College, Claremont, Calif. "La pcesia de Salomé Ureña de Henriquez".

Ångel Luis Morales, Universidad de Puerto Rico, "Puerta al tiempo en tres voces. Poema de Luis Palés Matos".

Alicia Perales Ojeda, Universidad Nacional de México, "La didáctica del español". 
Cesáreo Rosa-Nieves, Universidad de Puerto Rico, "Preludio al tema del modernismo" (Ciclo generacional: 1917-1921).

Arnulfo D. Trejo, Univ. of California Library, Los Angeles, "El acercamiento entre las Américas a través de la enseñanza y del escrito impreso".

John Van Horne, University of Illinois, "El valor de la Araucana".

INviTADOS DE HONOR: Marcel Bataillon, Arturo Capdevila, Waldo Frank, Rómulo Gallegos, Max Hentíquez Ureña, Juana de Ibarbourou, Julio Jiménez Rueda, Raimundo Lazo, Juan Marín, Cecilia Mireilles, Luis Monguió, Mariano Picón Salas, Alfredo Āngel Roggiano, Rogelio Sinán y Arturo Torres-Rioseco.

\section{ARGENTINA}

Nuevo Presidente de la Academia Argentina de Letras. Ha sido elegido el Dr. Mariano de Vedia y Mitre. Nacido en Buenos Aires en 1881, hijo de Nicolás de Vedia y Dolores Mitre, pertenece a una de las más ilustres familias patricias de la Argentina. Doctorado en Jurisprudencia en la Universidad de Buenos Aires, ha repartido su vida entre la cátedra, la historia y la investigación teórica del derecho constitucional argentino e internacional, la historia de las ideas políticas, la crítica y la traducción literaria. Sus traducciones de Shakes. peare, Shelley y otros autores ingleses le han dado justo prestigio en el mundo hispánico. Poeta él mismo y autor de una Apología del traductor (1935), sabe dar a sus versiones fidelidad y justo sentido poético. Como crítico e investigador literario se destacan sus libros Shelley y el amor (1925), Las alegorías de Salomé (1936), Los más grandes poetas ingleses (1952) y En torno del monólogo de Hamlet (1943). Como poeta se recuerdan sus libros Poemas de la fundación (1937) y Una dulce canción (1944). Como historiador e investigador del Derecho ha publicado más de veinte volúmenes. Es también miembro de la Academia Nacional de la Historia y de la Academia de Derecho y Ciencias Sociales.

Nuevos profesores en la Facultad de Filosofía y Letras de Buenos Aires. Fueron designados, después de los concursos de rigor, los siguientes profesores en la sección Letras: Enrique Anderson Imbert, Salvador Bucca, Julio Caillet-Bois, 
Augusto Raúl Cortazar, Fermín Estrella Gutiérrez, José María Moner Sans, Marcos A. Morínigo, Antonio Pagés Larraya y Guillermo de Torre. Todos ellos son personas de reconocida competencia en el ambiente profesoral argentino y con prestigio en las letras hispánicas.

A. S. C. U. A. La Asociación Cultural Argentina para Defensa y Superación de Mayo, que preside el pensador argentino Carlos Alberto Erro y que se caracterizó por su denodada lucha contra la dictadura peronista, ha tenido a su cargo la realización del Primer Congreso Argentino sobre Federalismo. ASCUA surgió en un grupo de estudiosos profesores, maestros e intelectuales argentinos y alberga en su seno a miembros de las distintas profesiones, representantes de la producción, empleados, obreros y estudiantes, todos ellos dentro de una línea de conducta humana que defiende la tradición democrática de Mayo frente al llamado "revisionismo histórico", contrario a dicha actitud. Los filósofos Francisco Romero y Vicente Fatone, el poeta Enrique Banchs, brigadier Angel M. Zuloaga, el señor José Santos Gollán, director del suplemento dominical de La Prensa y el señor José P. Barreiro, director del diario $E l$ Mundo, son entre otras figuras de prestigio, sus miembros integrantes.

Nuevo Presidente de la Sociedad Argentina de Escritores. Ha sido elegido el profesor José Luis Romero, ex-Rector interino de la Universidad de Buenos Aires, catedrático, miembro del Partido Socialista, historiador de las ideas políticas argentinas y excelente intérprete de la realidad social de su país, como lo prueban sus libros recientes: Argentina: Imágenes y perspectivas (1956) y la segunda edición de Las ideas polticas argentinas que acaba de hacer el Fondo de Cultura Económica de México. El Dr. Romero ha viajado recientemente a E. U. Participó en México en el Congreso por la Libertad de la Cultura y dio conferencias en Cuba.

La revista Sur complió veinticinco años de vida. Fundada en 1931 por Victoria Ocampo, que ha sido y sigue siendo su Directora-propietaria, ha sido un alto ejemplo de cultura. universal en la gran ciudad cosmopolita del Plata. Ha tenido asesores eminentes como los desaparecidos maestros Amado 
Alonso y Pedro Henríquez Ureña y ha dado cabida en sus páginas a firmas de renombre en todo el mundo, a la par que ha estimulado a los jóvenes valores argentinos.

Colegio Libre de Estudios Superiores. Esta institución de cultura democrática, clausurada por la dictadura peronista, ha vuelto a convertirse en el centro de la más alta cultura independiente del país. Tribuna de los mejores pensadores $\mathrm{y}$ artistas argentinos, es, puede decirse, la verdadera universidad libre de Argentina.

Instituto Popular de Conferencias. Tribuna perteneciente al diario La Prensa, reabrió sus ciclos culturales al ser devuelto el diario a su dueño, el Dr. Alberto Gainza Paz. Preside dicho Instituto el conocido escritor Dr. Arturo Capdevila.

Sociedad Hebraica Argentina. Desarrolló un importante ciclo de conferencias sobre temas argentinos y universales de palpitante actualidad, que estuvo a cargo de José Luis Romero, Risieri Frondizi y José Babini.

Ateneo Iberoamericano. Reinició sus actividades después de una clausura de varios años durante la dictadura, $y$ ha vuelto a ser el hogar de la confraternidad hispanoamericana en Buenos Aires.

Instituto Cultural Argentino-Norteamericano. Patrocinó un ciclo de conferencias del profesor Luther S. Mansfield. EI Agregado Cultural norteamericano en Buenos Aires, Dr. Chase, desarrolló un ciclo sobre la cultura norteamericana de este siglo.

Visita de escritores. Visitaron Buenos Aires y dieron conferencias en diversas instituciones, el novelista Arturo Barea, el escritor colombiano Germán Arciniegas, el poeta peruano Xavier Abril, el director de Cuaderni-Iberoamericani de Turín, Dr. G. M. Bertini y los escritores uruguayos Juvenal Ortiz Zaralegui y Emir Dodríguez Monegal, entre otros.

Congreso de Bibliotecarios. Presidido por el Presidente de la Comisión Argentina de Bibliotecarios, Sr. Germán García, se desarrolló el Primer Congreso de Bibliotecarios Argen- 
tinos, que tomó importantes decisiones de interés para la difusión del libro argentino.

Nuevo Director de la Biblioteca Nacional. Fue designado el prestigioso escritor Jorge Luis Borges, por demás conocido como para destacar sus méritos o el acierto de la designación.

Embajador en la UNESCO. Fue designado el novelista Eduardo Mallea, quien presidió también la representación argentina en la novena reunión de la Comisión de las Naciones Unidas para la Educación, la Ciencia y la Cultura, celebrada en Nueva Delhi. Integraron dicha misión el Sr. Director de Cultura del Ministerio de Educación, Prof. Julio Caillet-Bois, $\mathrm{y}$ el Rector de la Universidad Nacional del Sur, Prof. Vicente Fatone.

Instituto Argentino de Crítica Literaria. Presidido por el historiador Enrique de Gandía, rindió homenaje a tres escritores desaparecidos: el novelista español Pío Baroja, el poeta argentino Vicente Barbieri y el ensayista y narrador, también argentino, Pablo Rojas Paz.

Asociación Argentina de Críticos Literarios. Quedó constituida esta nueva entidad argentina con la intervención de los siguientes socios fundadores: Jorge Luis Borges, Carmen Gándara, Roberto F. Gusti, Eduardo González Lanuza, Arturo Morasso, Ezequiel Martínez Estrada, Luis Emilio Soto, Guillermo de Torre, Alfredo J. Weiss, etc.

Academia Internacional Rubén Dario. Acaba de fundarse y de elegir sus autoridades, presidida por Arturo Marasso, conocido especialista en Rubén Darío. Tendrá como fin el mantenimiento del culto al célebre poeta nicaragüense. Tratará de editar una publicación periódica que se llamará Revista Mundial.

Asociación Argentina por la Libertad de la Cultura. Rindió homenaje al filósofo Alejandro Korn, con motivo de cumplirse el vigésimo aniversario de su fallecimiento.

Nuevas casas editoras. Se han establecido nuevas casas editoras en distintos puntos del país. Destacamos entre las más recientes: El Litoral, en Santa Fe; Agora, en Buenos Ai- 
res, destinada a difundir libros de la historia, la vida y la cultura norteamericanas, y Editorial Reconstruir, que cuenta ya con una docena de títulos de autores del prestigio de Albert Camus, Herbert Read, Francisco Romero, Luis Franco, etc.

\section{Fallecimientos.}

En la ciudad de Buenos Aires, falleció el 11 de septiembre de 1956, el poeta y escritor don Vicente Barbieri, presidente de la Sociedad Argentina de Escritores. Era autor de varios libros de poemas, como: Fábula del corazón, 1939; Arbol total, 1940; Corazón del oeste, 1941; La columna y el viento, 1942, Premio Municipal de Poesía; Número impar, 1943; Anillo de sal, 1946; El bailarín, 1953, y de obras en prosa como El rio distante (Relatos de una infancia), 1945; Desenlace de Endimión, 1951. Había merecido también en 1946, el Premio Nacional de Poesía. A pocos días de su muerte fue puesta en escena su tragedia, Facundo en la ciudadela, en el Teatro Nacional Cervantes. (Sobre su obra puede consultarse, José Luis Ríos Patrón, Vicente Barbieri, Editorial La Mandrágora, Buenos Aires, 1954, con amplios detalles bibliográficos y críticos).

El $1^{\circ}$ de octubre falleció en Buenos Aires el escritor Pablo Rojas Paz, ensayista y novelista, con una gran labor periodística, siendo últimamente redactor de La Prensa. Era autor de Paisajes y meditaciones, 1924 ; La metáfora y el mundo, 1926; El perfil de nuestra expresión, 1929 ; Hombres grises, montañas azules, 1930, Hasta aquí, no más, 1936; El patio de la noche, 1940, etc.

\section{Revistas}

Bibliograma. Número 15, Director: Aristóbulo Echegaray.

Organo difusor del Instituto Amigos del Libro Argentino. Trae una entrevista a Enrique Anderson Imbert; artículos de Augusto Raúl Cortazar, Héctor Eandi, Dardo Cúneo, F. J. Solero, y una buena cantidad de críticas bibliográficas (julio y agosto). 
Ciudad. Número 4-5, Director: Carlos Manuel Muñiz.

Casi en su integridad el número está dedicado a Francisco Romero. (Los anteriores se ocuparon de Ezequiel Martínez Estrada y Jorge Luis Borges). Allí vemos: La concepcion antropológica de Francisco Romero, por Juan Carlos Torchia Estrada; La duatidad del hombre en Francisco Romero, por J. Kogan Albert; Persona y libertad en la filosofía de $F$. R., por Norberto Rodríguez Bustamante; La idea de cultura en la filosofía de F.R., por Raúl Alvarez Forn; Culturas de Oriente $y$ crisis occidental según $F$. R., por Víctor Massuh; Romero y las ideas lógicas, por Juan Bruera, y una Bibliografía de Francisco Romero, por Horacio Jorge Becco.

Comentario. Rev. trimestral, número 12, septiembre de 1956.

Publicación del Instituto Judío Argentino de Cultura e Información. En el presente figuran: Carlos Alberto Erro, $E l$ federalismo argentino en 1956; Sergio Bagú, Julián Martel y el reatismo argentino; Julio Arámburu, El rango civil del general Paz; David José Kohon, El guacamayo rojo (cuento), etc. Su importante sección crítica contiene reseñas de Martínez Estrada, Gregorio Weinberg, Attilio Dabini ,Raúl Galán, Antonio Pagés Larraya, Luis Ordaz, etc.

Ha aparecido el primer número de la revista "A", publicación de arte, con los auspicios de la Editorial Nueva Visión. En este número inicial encontramos poemas de Raúl Gustavo Aguirre y Edgar Bayle, con reproducciones de Sarah Grilo, Fernández Muro y Alfredo Hlito.

El número 22 de la revista "Poesía Buenos Aires", contiene una versión de Nosotros dos aún, poema de Henri Michaux, una carta de René Char, poemas de Wallace Stevens, Giusseppe Ungaretti, etc.

En Jujuy, provincia del norte argentino, circula ::Tarja", conducida por un grupo de elementos jóvenes, capitaneados por Jorge Calvetti.

En Buenos Aires aparece el primer número de T-A (Tiempo de América), con un material gráfico sorprendente y artículos como Psicopatología de la reacción en el arte, de Herbert Read; La adolescencia en crisis, por Jorge de Lima; 
Tupac-Amaru, el precursor, por Felipe Cossío del Pomar; Poetas músicos y cantores del antiguo Méjico, por Marcelino M. Román; Función social de la nueva poesia, por Rodolfo Alonso; García Lorca, por Francisco Urondo, y Roberto F. Giusti, por Luis Emilio Soto. Además una sección poética con trabajos de Antonio Esteban Agüero y Raúl Gustavo Aguirre. (Número uno, octubre de 1956, dirección, Cangallo 439, Oficina 509, Bs. As., Redacción, Danilo Romero).

Correspondencia. Número 2, octubre 1956. (Dirección de un comité, entre ellos Cécar Fernández Moreno, Eduardo Dessein, Ramio de Casasbellas, Jaime Alberto Barceló, etc.).

Se inicia con un trabajo inédito del poeta fallecido, don Baldomero Fernández Moreno, titulado Figuras del polvo y de la garúa (El meritorio Pérez); Realidad de la nueva poesía argentina, por Rubén Vela; Poemas de Jorge Carrol; una movida sección bibliográfica, clasificada como "La licuadora", donde la crítica desmenuza a los elegidos en forma muy humorística y mordaz. Casi todo su material está dedicado al grupo de jóvenes actuales, especialmente los poetas, que van surgiendo con una obra de importancia.

Ficción. Revista-libro bimestral, dirigida por Juan Goyanarte.

Número 3, septiembre-octubre de 1956.

Escriben: Luis Gudiño Kramer, Chamamé; Manuel Mújica Láinez, El retrato amarillo; Gloria Alcorta, La tortura perfecta; Anderson Imbert, El beso; Aristóbulo Echegaray, S. O. S. Música de Jazz; Luis Pico Estrada, Paraguay y Talcahuano; Luis Emilio Soto, Juan Maria Gutiérrez y la tradición del juicio literario; F. J. Soleto, Eugenio Cambaceres y la novela argentina; Pablo Rojas Paz, El centauro moribundo; Eduardo Dessein, Una fábula. Además una sección bibliográfica donde figuran E. Martínez Estrada, Carlos A. Loprete, Celia de Diego, Augusto Indarrega, Solero y otros. Esta revista-libro tiene 194 páginas. (Dirección: Paraguay 479, Buenos Aires). 
Noa. La revista "Movimiento NOA, 3", apareció en septiembre, bajo la dirección de Franco Di Segni. Entre sus artículos anotamos: Parā una novela de las tendencias artísticas de nuestro tiempo, por Ernesto B. Rodríguez; Música nueva, por Francisco Kröpfl; Henri Michaux: una actitud, por Osvaldo Svanascini; Miserable miracle, por Félix Gattegno, y varias notas sobre actividades plásticas en nuestro país y una lista de los participantes a la Bienal de Venecia.

El diario "Crítica" comienza a publicar un "suplemento literario", como sus colegas "La Prensa", "La Nación", "Clarín", etc. Las letras hispano-americanas están a cargo de $\mathrm{H}$. A. Murena y las mundiales a cargo de J. R. Wilcok. Este suplemento aparecerá semanalmente, todos los días miércoles.

Vigilia. Número 5-6. Director: Hugo Delfor Mangini.

Artículos de Karl Jaspers, Edgar Bayley, Raúl G. Aguirre, traducciones de Cesare Pavese y poemas de Hugo Gola, Carmen Bruna, etc.

\section{BOLIVIA}

Ministro de Educación. Fue designado el historiador de la literatura boliviana Fernando Díez de Medina. Nacido en La Paz en 1908, pertenece a la llamada "genración de la fe", la que, salida en 1935 del desastre del Chaco, tiene como propósito fundamental reconstruir a Bolivia. Desde 1948 a 1950 sostuvo el "Pachakutismo", grupo cívico renovador de tendencia vernácula. En 1950 obtuvo el Gran Premio Nacional de Literatura por su libro Nayjama. Es una de las figuras representativas del pensamiento boliviano actual y de la literatura hispanoamericana en general.

Fallecimiento de Franz Tamayo. En agosto de 1956 falleció en La Paz, a los 77 años de edad, Franz Tamayo, prominente hombre público y figura cumbre de las letras bolivianas. Nacido en 1879, se graduó de abogado, fue a perfeccionarse a París, formó parte desde 1913 del Partido Radical, fue diputado y electo Presidente de la República, cargo que no llegó 
a ocupar debido a un golpe de estado. Crítico erudito en sus estudios sobre Horacio y el arte lírico, poeta en La Prometheida y la Oceánica, su personalidad se destaca, sobre todo, como pensador. En este sentido se le compara con Vasconcelos, Vaz Ferreira, Francisco Romero y otros pensadoras de la América Hispánica. Proverbios y Creación de la pedagogía nacional son sus mejores obras como hombre de pensamiento. Scherzos y Odas son sus mejores creaciones líricas.

\section{COLOMBIA}

Baldomero Sanin Cano recibe honores. Su fallecimiento.

$\mathrm{Al}$ decano de las letras hispanoamericanas, al cumplir 95 años de edad, la Academia Colombiana de la Lengua, de la que era miembro, lo ascendió a "Miembro honorario". El 12 de mayo de 1957, a los 96 años de edad, muere en su ciudad natal. Véase nuestra nota necrológica en este mismo número.

Rivas Sacconi es nombrado Ministro. El lingüista e investigador de las letras colombianas, Dr. José Manuel Rivas Sacconi ha sido designado Ministro de Relaciones Exteriores. Latinista, sabio, filólogo, formado en Italia, doctor en Derecho $y$ en Letras, ex representante de Colombia en la UNESCO, llega al alto cargo a los 39 años de edad. Retiene su puesto de Director del Instituto Caro y Cuervo de filología e investigación literaria.

La hija de Guillermo Valencia, Ministro de Educación. La señora Josefina Valencia de Hubach, hija del poeta Guillermo Valencia, ha sido designada Ministro de Educación. Por primera vez en la vida institucional de Colombia, una mujer ocupa una cartera ministerial.

\section{CUBA}

Nuevo Director del Archivo Nacional. Fue designado el Dr. Félix Lizaso, ex Director de Cultura, ensayista e historiador, director también de los Archivos de José Martí. 
Instituto Nacional de Cultura. Es el nuevo nombre que tiene ahora la ex Dirección Nacional de Cultura. Tiene como órgano de difusión un Boletín Informativo, que dirige la Dra. Marilyn Ichaso.

Visita de escritores. Visitaron Cuba, en donde dieron conferencias, el prestigioso crítico hispano-argentino Guillermo de Torre y el conocido historiador de la literatura hispanoamericana y del Perú, Dr. Luis Alberto Sánchez, actual catedrático de la Universidad de San Marcos de Lima.

Centro de Altos Estudios. Quedó inaugurado en La Habana, patrocinado por el Instituto Nacional de Cultura. En dicha oportunidad habló el Director del mencionado Instituto, Dr. Guillermo de Zéndegui.

Patronato del Teatro. Otorgó el Gran Premio en el concurso anual "Luis de Soto" al autor dramático cubano José Enrique Montoro Agüero por su obra Desviadero 23.

Salvador de Madariaga, en Cuba. El eminente escritor español tri-lingüe habló sobre "La angustia de la libërtad", en el Lyceum Lawin Tennis Club de La Habana.

Ciro Alegría, en la Sociedad Lyceum. El eminente novelista de El mundo es ancho y ajeno dio un ciclo de conferencias er la Sociedad Lyceum, en el que trató diversos aspectos de la creación narrativa.

Academia Municipal de Artes Dramáticas. En esta institución tuvo a su cargo un Ciclo Teatral el escritor Salvador Bueno.

Julián Marías, en el Instituto Nacional de Cultura. El conocido pensador español visitó la isla, de regreso de los EE. UU. y en viaje a España y pronunció conferencias en la institución citada.

Comisión Cubana de la UNESCO. Invitó a dar conferencias al crítico chileno Ricardo Latcham.

Colegio de Ciencias y Filosofía y Letras. Jorge Mañach ofreció un curso sobre problemas de la educación secundaria en Cuba.

Ateneo de Matanzas. Dio conferencias el Dr. Max Henríquez Ureña.

Revista Cubana de Filosofía. Publicó un número (enerojunio de 1956) en homenaje a Ortega y Gasset. Se abre con 
una nota editorial escrita por el Director de la Revista, Dr. Humberto Piñera Llera.

Ateneo de La Habana. El escritor Alberto Baeza Flores hizo una importante exposición crítico-histórica sobre las revistas cubanas del siglo XIX.

Cosme de la Torriente.

A la edad de 84 años falleció el patriota cubano Cosme de la Torriente y Peraza, soldado, diplomático, abogado y estadista. Era el Presidente de la Comisión Cubana de la UNESCO. Cosme de la Torriente y Peraza nació el 27 de junio de 1872 en la estancia de su padre, La Isabel, situada cerca de Jovellanos, en la provincia de Matanzas. Después de cursar bachillerato en el Instituto de la capital de esa provincia, estudió abogacía en la Universidad de La Habana, hasta 1895, año en que se alistó en la revolución estallada entonces. Ya en 1892 había obtenido el título de licenciado en filosofía, profesión que estudiaba juntamente con la de abogacia, y, a su regreso del campo de batalla, en 1898, se graduó como doctor en leyes.

Durante la revolución de 1895 se dirigió a Estados Unidos para organizar fuerzas expedicionarias destinadas a emancipar a su país, y debido a esas actividades fue encarcelado en distintas ocasiones en Washington, Delaware, Nassau -islas Bahamas-y Nueva York. Finalmente, participó en la famosa expedición del vapor Bermuda, a las órdenes del general Calixto García, que desembarcó con éxito en Baracoa, y durante el resto de la guerra sirvió con los generales Máximo Gómez, José Miaría Rodríguez, Francisco Carrillo y José Manuel Capote.

Desde 1906 se dedicó al ejercicio de la profesión y a los asuntos públicos. A su regreso de España fundó y organizó e] Partido Conservador Nacional, llegando a ser presidente de esa agrupación en 1914. En abril de 1918 fue elegido senador y más tarde Secretario de Estado en el gobierno del Presidente Menocal. Otros cargos que desempeñó fueron: embajador en Washington, 1923-1925; Presidente de la Cuarta Asamblea de la Liga de las Naciones (1923), de la Asociación Cubana de las Naciones Unidas, de la Sociedad Cubana de Derecho Internacional y de la Comisión Nacional Cubana de la UNESCO ; Vicepresidente de la Academia Cubana de la Histo- 
ria ; miembro correspondiente honorario de la Sociedad Geográfica de Madrid y miembro correspondiente de la Real Academia Hispano-Americana de Ciencias y Artes de Madrid.

Torriente publicó muchos libros sobre política y jurisprudencia internacional, así como sobre asuntos relacionados con la gesta de la independencia cubana y la ulterior reconstrucción nacional.

\section{CHILE}

\section{Juan Marín, en la Unión Panamericana}

El doctor Juan Marín, miembro distinguido de nuestro Instituto, acaba de ser nombrado Director del Departamento de Asuntos Culturales de la Unión Panamericana en reemplazode nuestro estimado compañero Erico Verissimo, quien regresa al Brasil después de servir en ese cargo durante varios años. El doctor Marín tomó parte muy activa en el último Congreso del Instituto celebrado en Berkeley el año 1955. Conocido de sobra como escritor, Juan Marín trae a la Unión Panamericana, además, su rica experiencia de diplomático y director de sociedades literarias en Chile. Sus colaboraciones periodísticas aparecen a menudo en diarios tan diversos como El Mercurio, de Santiago; El Diario Latino, del Salvador; El Nacional, de México, etc., etc. No hace mucho tiempo la editorial chilena Zig-Zag publicó su último libro, El Egipto de los faraones, que se convirtió rápidamente en un best-seller y que recibió el Premio Atenea auspiciado por la Universidad de Concepción. Juan Marín es autor, también, de novelas y cuentos como Paralelo 53, Sur, Naufragio y Cuentos de viento y agua. Nuestro Instituto le da la bienvenida a los EE. UU. y le hace llegar sus deseos de que su obra cultural se vea coronada con un éxito completo.

Nuevo académico

Ricardo A. Latcham ha sido nombrado miembro de la Academia Chilena, correspondiente de la Real Española, en sesión de 20 de agosto de 1956. Se le conoce como crítico literario, biógrafo y catedrático de Literatura Hispanoameri- 
cana en el Instituto Pedagógico de la Universidad de Chile. En la Academia le tocará reemplazar a Misael Correa Pastene, periodista, crítico literario y cuentista, fallecido a comienzos de 1956.

El núm. 370 de Atenea, correspondiente a los meses mayo y junio de 1956, con un otal de más de doscientas ochenta páginas de texto, aparece dedicado a estudios sobre Mariano Latorre, con motivo de su reciente fallecimiento. Colaboran Milton Rossel, Ricardo A. Latcham, Raúl Silva Castro, Juan Uribe-Echevarría, Luis Merino Reyes, Homero Castillo y otros escritores.

Manuel Rojas obtuvo las palmas como uno de los primeros novelistas chilenos contemporáneos con la publicación de Hijo de ladrón (Nascimento, 1951). Después de varias ediciones en español, la obra ha sido, además, traducida al inglés y al alemán. La primera de esas versiones lleva el título de Born Guilty, y ha sido publicada por Library Publishers, de Nueva York, en versión que firma Frank Gaynor. La segunda, vertida por el Dr. A. M. Rothbauer, se titula Wartet, ich komme mit, y ha sido editada en Austria por la casa editorial Styria. Ambos libros son de 1955.

Alberto Ried, compañero de Pedro Prado y de Juan Francisco González en la aventura del grupo artístico Los Diez, que floreció en Santiago de Chile en 1916, ha publicado una serie de capítulos autobiográficos en su libro El mar trajo mi sangre (Editorial del Pacífico, 1956). Fuera de los dos personajes ya mencionados, en el libro aparecen útiles referencias a no pocos otros chilenos distinguidos en la primera mitad del siglo $\mathrm{xx}$.

Gabriel Sanhueza acaba de publicar con el título de Santiago Arcos, comunista, millonario y calavera (Editorial del Pacífico, 1956), una biografía de aquel personaje. Fundó en 1850 la Sociedad de la Igualdad, que tiene sitio propio en la historia política de Chile como el primer partido proletario, y aunque vivió poco tiempo en su patria, fue compañero y amigo de sus escritores y periodistas. La biografía, que se debe a Sanhueza, está muy bien informada y escrita con elegancia.

Hernán Poblete Varas traza en su Misión en el Pacífico (Editorial del Pacífico, 1956) un amenísimo relato del viaje 
desde Valparaíso hasta el Japón, pasando por las islas oceánicas, y con vuelta por los Estados Unidos. Escrito con buen estilo, es un libro de grata lectura hasta para quienes no sienten demasiado el hechizo de los viajes contados por otros.

Francisco Coloane, distinguido ya en la literatura chilena como autor de relatos sobre la ruda vida en las regiones más australes del país (Magallanes, los archipiélagos y canales), ha reunido ocho de sus cuentos en Tierra del Fuego (Editorial del Pacífico, 1956). Han sido muy bien recibidos por la crítica literaria.

Páginas olvidadas y dispersas de Alberto Blest Gana (1830-1920) aparecen en El jefe de la familia, que acaba de publicar la Empresa Editorial Zig-Zag. El título corresponde a la única pieza dramática que se conoce del autor, reputado como el mejor novelista chileno de todos los tiempos; siguen artículos de costumbres y dos estudios sobre literatura, en que el autor fija sus ideales estéticos. La recopilación se debe a Raúl Silva Castro, que en 1941 publicó una completa biografía de Blest Gana, premiada por la Universidad de Chile.

Centenario de Menéndez y Pelayo. El programa de celebración del centenario de este escritor español se abrió para Chile con la serie de conferencias que auspiciaba el Departamneto de Extensión Cultural de la Universidad Católica. En dicha serie se pronunciaron las siguientes conferencias:

- Menéndez Pelayo y la tradición, por el R. P. Osvaldo Lira, 15 de octubre.

-Menéndez Pelayo al través de su correspondencia, por Roque Esteban Scarpa, 16 de octubre.

-Menéndez Pelayo y Chile, por Raúl Silva Castro, 17 de octubre.

-Menéndez Pelayo, crítico, por Fernando Durán Villarreal, 18 de octubre.

La Biblioteca Nacional quiso asociarse, igualmente, al centenario de Menéndez y Pelayo y organizó una exposición de los libros del maestro que guarda en sus colecciones.

La exposición fue inaugurada el 2 de noviembre con una ceremonia a la cual asistieron el Ministro de Educación Pública, representantes de la Embajada de España y otras autoridades. Inició el acto el Director General de Bibliotecas, 
don Eduardo Barrios, con un breve discurso alusivo, y en seguida pronunció una conferencia sobre Menéndez Pelayo y la exposición de sus obras don Raúl Silva Castro. La exposición ha permanecido abierta hasta el día 17 de noviembre. Se publicó catálogo impreso de la misma.

La Academia Chilena, correspondiente de la Real Española, llevó a cabo una velada en el mismo local el día 23 de noviembre, en la cual hicieron uso de la palabra don Pedro Lira Urquieta y don Ricardo A. Latcham.

\section{EL SALVADOR}

Muere Francisco A. Gavidia (1864-1955). Con la muerte de Francisco A. Gavidia desaparece el que fuera decano de las letras salvadoreñas. Su obra, como lo recuerda Rubén Darío en su Autobiografía, está directamente vinculada a las renovaciones métricas modernistas del autor de $A z u l$.

\section{MEXICO}

Congreso por la Libertad de la Cultura. Celebróse en la capital mexicana, con la asistencia de las más destacadas figuras del mundo cultural de nuestro tiempo en el hemisferio occidental. Fue presidido por Salvador de Madariaga y contó con la asistencia de delegaciones de casi todos los países de habla hispánica.

\section{Nuevas revistas}

Acaban de aparecer las revistas Panoramas y la Revista. de Bellas Artes, la primera publicada por las Galerías Excélsior y la segunda por la Secretaría de Educación Pública.

México en la Cultura. Nueva publicación, en un volumen, de la sección literaria del periódico Novedades, de la ciudad de México.

Premio. El premio Ruiz de Alarcón para 1956 lo ganó Luis G. Basurto por su comedia Miércoles de ceniza; el segun- 
do puesto lo obtuvo Humberto Robles Arenas con su obra Los desarraigados, que ya había merecido el premio del periódico El Nacional.

Berta Gamboa de Camino (1888-1957). El $1^{\circ}$ de enero murió la profesora y esposa del poeta español León Felipe Camino. La señora Gamboa de Camino se especializaba en la literatura de la Revolución mexicana. Dictó clases sobre esta materia por varios años en la Escuela de Verano de la Universidad Nacional Autónoma.

Nuevos académicos. El 31 de octubre de 1956 ingresó en la Academia Mexicana de la Lengua Española el Dr. Jesús Guisa y Azevedo, como individuo de número. El 17 del mismo mes había ingresado el profesor Jesús Silva Herzog, director de la revista Cuadernos Americanos, y el 11 de junio el Dr. Luis Garrido. El 24 de febrero había sido aceptado el escritor Octaviano Valdés.

Diploma. El Dr. Francisco Monterde, en nombre de la Academia Mexicana de la Lengua Española, entregó a la escritora doña María Enriqueta Camarillo de Pereyra un diploma -en función celebrada el 17 de octubre de 1956-como reconocimiento a quien ha usado la lengua española con gran decoro.

Manuel Toussaint (1895-1955) falleció en Nueva York el 22 de noviembre; destacó en la crítica literaria y las artes plásticas.

Alfonso Cravioto (1884-1955), poeta y crítico, falleció en la ciudad de México el 11 de septiembre. Desde muy joven se dedicó a las letras, formando parte del grupo literario de la Revista Moderna y, poco después, del Ateneo de la Juventud.

\section{PUERTO RICO}

Federico de Onís ha renunciado a la Universidad de Puerto Rico, en la cual era decano del Departamento de Español. El ilustre crítico español que tanto ha hecho por las letras hispanoamericanas, fue durante muchos años director del Hispanic Institute de la Universidad de Columbia, en Nueva York. Ahora pasará a ocupar un nuevo cargo en la Universidad de 
Santa Clara, en Cuba, en donde piensa establecerse, después de un viaje a la Argentina, según nos manifiesta por carta. Auguramos al sabio historiador y antologista de la poesía hispanoamericana el mejor de los éxitos en su futura labor, que honrará, como en casos anteriores, al país que lo acoge y a todo el mundo hispánico. 